\title{
Health-Seeking Behaviors and Health Information Gathering in \\ Older Mexican American Males
}

Evangeline M. Ortiz-Dowling, $\mathrm{PhD}^{1}$, Cheryl Der Ananian, $\mathrm{PhD}^{2}$, Linda Larkey, $\mathrm{PhD}^{2}$, Steven $\mathrm{P}$. Hooker, $\mathrm{PhD}^{2}$

Corresponding author:

Evangeline M. Ortiz-Dowling, $\mathrm{PhD}, \mathrm{RN}$

College of Nursing

The University of Arizona

1305 N. Martin Avenue

P.O. Box 210203

Tucson, Arizona 85721-0203

Tel: (520) 256-2808

Fax: (520) 626-4062

Email: dowling@email.arizona.edu

First author:

Evangeline M. Ortiz-Dowling, $\mathrm{PhD}, \mathrm{RN}$

College of Nursing

The University of Arizona

1305 N. Martin Avenue

P.O. Box 210203

Tucson, Arizona 85721-0203

Tel: (520) 256-2808

Fax: (520) 626-4062

dowling@email.arizona.edu

Second co-author:

Linda K. Larkey, PhD

College of Nursing and Health Innovation

Arizona State University

500 N. $3^{\text {rd }}$ Street

Phoenix, AZ 85004

Linda.larkey@asu.edu

Third co-author:

Cheryl Der Ananian, PhD

College of Health Solutions

Arizona State University

500 N. $3^{\text {rd }}$ Street

Phoenix, AZ 85004

Cheryl.derananian@asu.edu

Fourth co-author:

Steven P. Hooker, PhD

School of Nutrition and Health Promotion 
Arizona State University 550 N. $3^{\text {rd }}$ Street

Phoenix, Arizona 85004

Tel: (602) 496-1727

Fax: (602) 496-0544

steven.hooker@asu.edu 


\begin{abstract}
Evidence suggests that men's health disparities are linked to attitudes and beliefs toward health promotion and risk reduction, participation in high-risk behaviors, and limited health care access and utilization influenced by socioeconomic challenges. However, we know less about the specific factors related to health disparities in older Mexican American males. The purpose of this study was to explore the cultural, social, environmental, and gender factors influencing health-seeking behaviors and health information gathering in older Mexican American males. Indepth interviews were conducted with 14 older Mexican American males aged 65-80 years. Based on language preference, interviews were conducted in either Spanish or English. A standardized moderator's guide was used to examine the participant's perceptions of health, maintenance of health, gender influences on health, and sources of men's health information and health promotion programs. Data were collected, organized, and analyzed following the methodology of transcendental phenomenology and the Social Ecological Model. Two major themes related to health-seeking behaviors and health information gathering emerged: 1) top external sources heeded include women, health care providers, and the Internet and 2) men regard inner prompts for self-determined self-care. Future research should consider these external sources and inner prompts when developing targeted health promotion interventions, such as physical activity programs, with older Mexican American males.
\end{abstract}

Keywords: men's health, social ecological model, health promotion, phenomenology, social determinants of health, gerontology, health disparities, healthy aging, culture 
The life expectancy of men is reported to be five years younger than women (National Center for Health Statistics [NCHS], 2012). Globally, for every 100 women aged $\geq 65$ there are only 84 men, and for every 100 women aged $\geq 80,61$ men (United Nations Population Fund [UNFPA], 2012). The leading cause of death in men is heart disease, accounting for one in every four male deaths (Centers for Disease Control and Prevention [CDC], 2013a). However, men also have higher death rates for malignant neoplasms, unintentional injuries, diabetes mellitus, and suicide (Baker, 2018; NCHS, 2012). In 2016, type two diabetes prevalence were higher in older men than in older women (Nordstrom, A., Hadrevi, Ollsson, Franks, \& Nordstrom, P., 2016). Older Mexican American (MA) men, aged 60 - 69 years, also had a higher proportion of undiagnosed diabetes than MA women (7.7\% versus 1.4\%, respectively) (Salinas, 2011, p. 335). Among MA men in general, diabetes is associated with older age (Watt et al., 2016). It is reported that the total indirect costs (i.e., lower worker productivity secondary to illness and premature death) which men's health disparities impose on our society for a four-year period is \$436 billion (Thorpe, Richard, Bowie, LaVeist, \& Gaskin, 2013). It is believed that healthier men would decrease the economic costs of lost productivity (Baker, 2018, p. 147; Leone, Rovito, Mullin, Mohammed, \& Lee, 2017).

Men's attitudes and behaviors may contribute to their risk for injury, disease, and death. Compared to women, men use more alcohol, tobacco, and drugs (Baker, 2018; Courtenay, 2011; Gorman, Read, \& Krueger, 2010; Houle et al., 2017; Stimpson \& Peek, 2005). In fact, heavy drinking and related problems are highest among Hispanic men compared to men of other racial and ethnic groups in the United States (US) (Field et al., 2015, p. 1). Men also engage in more reckless driving and drive drunk more often than women. Promiscuity and high-risk physical 
activities (e.g., dangerous sports, physical fights, and gun use) also undermine the health of men and those around them (Courtenay, 2011).

The under use of health care services (e.g., preventive care) by men has also been identified as a global problem (Baker, 2019; Leone et al., 2017). This phenomenon has been linked to culture and gender role structures (Leone et al. 2017). Perkins, Hazuda, \& Cortez (2004 \& 2006) found that older MA males feared harm and disempowerment from a lack of control and knowledge of the health care system. Gender, particularly male sex, was found to influence health care decision-making in older MA men more than other variables, such as religion (Espino et al., 2010).

Among health care providers, men's health mostly involves sexual dysfunction, testicular cancer, and prostate health. There is a lack of knowledge among health care professionals on how to effectively engage men to promote health and reduce risk (Baker, 2018). The utilization of primary care services for prevention and screening has the potential to identify disease at an early age, increase chances of survival, decrease costs associated with illness, and improve quality of life (Ailinger, 1989; Noone \& Stephens, 2008; Peak, Gast, \& Ahlstrom, 2010; Sobralske, 2006), but the perceptions of and reasons for seeking health care among older MA men are not well known.

Social networks and support are associated with longevity. Unfortunately, men have much smaller social networks and fewer friends compared to women (Courtenay, 2011). Previous research has identified that low social support leads to an increased risk of mortality in older MA men (Hill, Uchino, Eckhardt, \& Angel, 2016). It is estimated that one-half of all men's deaths in the US each year could be prevented through lifestyle changes (Courtenay, 2011). 
Migration should also be considered when assessing health disparities among Hispanics (Olsen \& Anderson, 2018). More than 34\% of the Hispanics living in the US are foreign born (Zong, Betalova, \& Hallock, 2018). Immigrants are reported to have better health outcomes than their counterparts born in the US. Sadly, the more time an immigrant spends in the United States, the unhealthier the individual becomes (Gorman, Read, \& Krueger, 2010; IOM, 2009). Masel, Rudkin, \& Peek (2006) found significant associations between health behaviors and acculturation in the older adult MA population (mean age 72.5 years). As English language use and Anglo-American contact subscale scores increased, participants were more likely to be current or former smokers and drinkers than being a nonsmoker or lifetime abstainer (p. 692 \& 695). Driving factors behind this phenomenon are unclear; however, understanding it and developing interventions to reverse it are necessary (Gorman, Read, \& Krueger, 2010).

MAs are the largest and fastest growing population in the US with unique cultural, political, geographical, and historical characteristics (Fenelon, Chinn, \& Anderson, 2017; Garcia, M., Garcia, C., Chiu, Raji, \& Markides, 2018; Lampe, 2013). Unfortunately, data specific to older MA men's health disparities is scarce. The lack of evidence results from Hispanic populations often being generalized and represented as a homogenous group (CDC, 2013; Fenelon, Chinn, \& Anderson, 2017; Gold, 2014). Consequently, background literature for this study had to be extrapolated from research involving older MA adults in general. Insufficient data on race, ethnicity, gender, and language decreases the chance of properly addressing population-specific health disparities (Institute of Medicine [IOM], 2009). As a matter of fact, a male Hispanic participant in Gilbert, Barrington, Rhodes, \& Eng's (2016) study described frustration over the lack of recognition of the diversity in Hispanic subgroups. This participant expressed feelings of being a minority within a minority (p. 519). Garcia, M., Garcia, C., Chiu, 
Raji, \& Markides' (2018) study is reported to be "one of the first attempts in documenting the heterogeneity among older Hispanics by nativity, country of origin, and gender in life expectancies with morbidity and without morbidity" (p. 3).

Older MA males are an understudied group. Health promotion targeting this population of older men holds potential for addressing behavioral risk factors associated with higher risk for morbidities and mortality, but little is known about what sorts of health promotion sources might be most heeded. A man's identity and gender role develop as he interacts within his culture. Researchers have given little thought to how those cultural beliefs, values, worldviews, and life experiences influences a male's health care behaviors (Sobralske, 2006). Determinants of health should be examined in a broader view with consideration to factors such as culture, social support networks, social and physical environments, gender, and age Adler et al. (2016) . Therefore, the purpose of this study was to explore the cultural, social, environmental, and gender factors influencing health-seeking behaviors and health information gathering in older MA males.

\section{Theoretical Framework}

One approach to exploring the cultural, social, environmental, and gender factors influencing health-seeking behaviors and health information gathering in older MA males is through the Social Ecological Model (SEM). Ecology refers to the interrelationship between an organism and their environment (Sallis, Owen, \& Fisher, 2008). The core principles of ecological models propose that: 1) specific health behaviors have various influences and those influences interact across multiple levels; 2) ecological models should be behavior specific, and should identify the most significant influences at each level; and 3) interventions guided by the principles of an ecological model should consider all levels of influence in order to be most 
effective (Sallis et al., 2008; Stokols, 1992). Examining health behaviors at multiple levels (i.e., intrapersonal, interpersonal, institutional, community, and public policy) prevents researchers from focusing in on a single aspect of influence by providing a thorough picture of the relations between various levels of influence.

\section{Method}

This study was designed as a transcendental phenomenological qualitative inquiry into the cultural, social, environmental, and gender factors influencing health-seeking behaviors and health information gathering in older MA males (Moustakas, 1994). Clark Moustakas transcendental phenomenology focuses less on the interpretations of the researcher and more on describing the experiences of the individual (Creswell, 2013). The design implies moving beyond the routine and perceiving everything freshly, while being directed towards the source of meaning of lived experiences (Moustakas, 1994).

\section{Participants and Setting}

Eligible participants were male, $\geq 65$ years of age, self-identified as MA, able to comprehend and adequately respond to questions, and willing to participate in an audiotaped one-on-one interview. Purposive sampling was used to identify participants that were representative of the study population. Recruitment was supplemented through snowball sampling which involved asking early participants to refer other study participants (Polit \& Beck, 2017). Specifically, participants were recruited by word-of-mouth through organizations serving primarily community-dwelling ambulatory individuals which the corresponding author volunteered in (e.g., community associations). Potential participants were also referred by other study participants. Participants were stratified (i.e., seven per strata) by language preference or capability (e.g., monolingual Spanish versus bilingual Spanish) to enhance the representativeness 
of the participants and as a potential marker of the effects of acculturation or language on factors influencing health-seeking behaviors and health information gathering.

Recruitment scripts in English or Spanish were used to explain the research to potential participants. When an older MA male expressed interest in the study, the potential participant provided their contact information and was phoned by the corresponding author. The potential participant was then presented with four questions to screen for eligibility: 1) How old are you?

2) Are you of Mexican descent? 3) Are you Spanish-speaking only or are you bilingual? 4) Would you be interested in spending an hour this week sharing your thoughts on men's health promotion? If the older MA male met the inclusion criteria, then the potential participant was scheduled for an interview. At the time of the interview, the potential participant was consented and enrolled in the study prior to participating in the interview. Recruitment continued for four weeks, the amount of time needed to fill the language inclusion strata. Seventeen older MA men were contacted and 14 expressed interest and participated in this study. Study materials were available in English and Spanish and approved by the Institutional Review Board (IRB) at Arizona State University (ASU). All interviews were conducted in a private room in the participants' home or a community facility (e.g., private library study room). Two bilingual Hispanic females who have experience in qualitative research methodologies led the interviews.

\section{Participant Characteristics}

Study participants $(n=14)$ ranged in age from $65-80$ years, with a mean age of 71.2 years $(S D=4.5$ years $)$. In general, the participants were married, well-educated, mostly English speakers and readers at home, retired, and earned $>\$ 25,000$ annually (Table 2). 


\section{Measures}

Each participant's demographic data were collected after consent and prior to the interview using a 13-item self-report questionnaire that captured age, household living status, marital status, education, language, work status, and income (McEwen \& Murdaugh, 2012).

Information about acculturation was measured using the Acculturation Scale for Mexican Americans-II (ARSMA-II). The ARSMA-II is a 30-item Likert type scale that measures acculturation along three factors: language, ethnic identity, and ethnic interaction. The instrument factors into two scales: an Anglo Orientation Subscale (AOS) consisting of 13-items and the Mexican Orientation Subscale (MOS) containing 17-items. Both subscales were found to have good internal reliability with Cronbach's alphas of .86 and .88 for the AOS and MOS, respectively (Cuellar, Arnold, \& Maldonado, 1995).

Acculturation scores using the ARSMA-II are determined by subtracting the MOS mean from the AOS mean. The acculturation score is then compared to a set of cutting scores to determine the participant's acculturation level. The five levels within the cutting scores range from Level 1 (i.e., very Mexican oriented) to Level 5 (i.e., very assimilated) (Cuellar, Arnold, \& Maldonado, 1995).

\section{ARSMA-II}

The means and $S D$ for each of the ARSMA-II subscales are reported in Table 2. Using the established ARSMA-II acculturation levels (Cuellar, Arnold, \& Maldonado, 1995), 14.2\% of the participants were classified as Level 1 (i.e., very Mexican oriented), $42.9 \%$ were classified as Level II (i.e., Mexican oriented to approximately balanced bicultural), and $42.9 \%$ were classified as Level III (i.e., slightly Anglo oriented bicultural). Although participants were stratified by language preference or capability during the screening process, $78.6 \%$ of the participants 
reported speaking and reading mostly English at home. Interestingly, four of the monolingual Spanish speaking participants also attempted to reply to the interview questions in English even though the questions were presented in Spanish by fluent Spanish speaking MA investigators.

\section{Data Collection}

Audiotaped one-on-one interviews were conducted using a standardized moderator's guide (Table 1). This guide was adapted from an interview guide previously used in a health promotion study with older African American (AA) men (Friedman, Hooker, Wilcox, Burroughs, \& Rheaume, 2012). The standardized moderator's guide was grounded in the SEM (i.e., included questions representative of the levels of influence) and used during the interviews to ensure uniformity in data collection. If the participant did not understand a specific question, the PI used follow-up prompts to clarify the meaning and elicit a meaningful response. The interviews lasted 60-75 minutes, with breaks taken as needed by the participant. Data saturation was determined during ongoing data analysis when interviews no longer added to the findings (Patton, 2002). Corroboration amongst the principal investigator and three collaborators guaranteed that an adequate sample was interviewed.

\section{Data Analysis}

The audiotaped interviews were transcribed verbatim. Spanish transcripts were translated to English and verified for accuracy by the researchers and a Spanish translator. Moustakas' transcendental phenomenology provided the systematic approach to data analysis, the construction of the textual and structural descriptions of the studied phenomenon, and guided the identification of the essence of health as experienced by older MA males. NVivo for Mac software, version 10.2.1, organized the data. Data analysis began with horizonalization (i.e., approaching all data as being equal in value) (Moustakas, 1994). All investigators read the data 
and general thoughts were written in the margins of the transcripts. The researchers read the transcripts again, one-by-one, and line-by-line. Horizons (i.e., meaning units) were then identified and labeled as major horizons, unique horizons, and leftovers (Creswell, 2009; Moustakas, 1994). Codes were developed by abbreviating the horizons. These codes were then highlighted on the corresponding area of the transcript. Once the codes were agreed upon, they were conceptualized into themes based on processes, perspectives, contexts, relationships, and social structures. Themes were then labeled with textural (i.e., nature and meaning) and structural descriptions (i.e., factors accounting for the experience). Structural descriptions are 'the 'how' that speaks to conditions that illuminate the 'what' (texture) of experience. How (structure) did the experience of the phenomenon come to what (texture) it is?" (Moustakas, 1994, p. 98). The final step in analysis was the integration of the textural and structural descriptions into a descriptive statement of the essence of the phenomenon. Synthesis of the textural-structural descriptions represents the essence of the phenomenon at this current place and time (Moustakas, 1994).

Study credibility was addressed through member checking (Creswell, 2009). Following data analysis, the identified themes and essence statement were presented to five study participants who agreed to participate in a follow-up focus group. This focus group provided an opportunity to confirm or adjust the identified themes and essence statement.

\section{Results}

\section{Emerging Themes}

Two major themes regarding health-seeking behaviors and health information gathering emerged: 1) top external sources heeded include women, health care providers, and the Internet 
and 2) men regard inner prompts for self-determined self-care. Tables 3 and 4 contain select quotes that provide contextual illustration of the emerging themes.

\section{Top external sources heeded include women, health care providers, and the}

Internet. These major horizons were identified when the participants were asked the general health questions from the Moderator's Guide (Table 1). One man commented:

"I get my information through my wife because she is always up-to-date on everything that is going on. She asks, 'Did you hear about that? She has always been there telling me what I can and cannot do."

Another participant stated: "Once in a while I will talk to my wife about my health. My wife is very important in my health and health decisions. She is my constant companion. She is important to my health."

Wives were described as motivators for participation in future programs focused on health promotion in older MA males. One of the men shared:

"I would say that even creating programs with the wives. They have been your partners for life. You depend on each other. I think it may be more successful."

Participants described their wives as being healthy and as someone who practiced what she preached, lived a healthy lifestyle, had a good diet, was physically healthy, was mentally healthy, worked daily towards health, was spiritual, was calm, exercised daily, had a positive attitude, was a role model and constant support and education to her family and husband. A 72year-old participant stated:

"My wife, I would most consider the healthiest one. She practices everything she preaches. She is very committed, in terms of her health, her physical health, her mental health. Her diet has also influenced my diet." 
Another participant declared: "I would probably say my wife is very healthy. She watches what she eats, tries to eat healthy food, attempts to maintain regular physical exercise, and always watches me to make sure that I'm kind of the same boat with her; also, by making sure that I maintain my doctor's appointments. She is always aware if something may be ailing me. If I need to see a doctor or not."

Participants also stated that their wives prepare most of the meals; therefore, maintains a healthy diet for the entire family. Participants also indicated that their wives scheduled their doctor appointments and are credited for helping many of them maintain a regular physical activity (PA) routine by either verbally encouraging PA or participating in PA with them.

One participant shared: "My wife helps me the most. She tells me what I should be doing, too. She cooks me the food I should be eating. It's healthy food, like my doctor said. She also puts me to work! She motivates me to get up and move. There is a lot to do at our house. She also tells me to exercise, so that I can be healthy."

Health care providers. Participants reported a good relationship with their doctors. Doctors were reported to be a highly trusted source for health information. Participants stated that their doctor encouraged regular PA. One participant mentioned that his doctor stays current in medical research and often discusses new treatments and medications.

"My doctor is up-to-date on all this stuff. As a matter of fact, he wanted to give me a new medication, and I went to the base to get it. They didn't even have it yet. It was too new. I trust him. I have known him for more than 15 years."

Internet. Participants stated they gathered their health information from online sources. One participant stated: "It's funny, but now I go to the Internet just like the kids do." Participants 
stated that although they gather a lot of health information online, they always consult their doctor before implementing a remedy.

Composite textural description. Wives, health care providers, and the Internet are integral factors in encouraging this population to engage in health promoting behaviors. Many of the participants and their wives engage in health promoting behaviors together. Wives are credited for providing healthy meals and maintaining health care appointments for the family. Physicians are a trusted source for health information and encourage health promoting behaviors.

Composite structural description. Motivators for health promoting behaviors come from knowledge that was gained mostly through discussions with their wives and doctors. The participants also referred to the Internet for information regarding health and wellness. Wives were described as health promoters and considered a vital component to health care decisionmaking. Physicians were always referred to before adopting and implementing health information gathered off the Internet.

Textural-structural synthesis. The participants in this study refer to their wives, physician, and Internet for information on health and wellness. These sources were described as motivators to engaging in health promoting behaviors.

Men regard inner prompts for self-determined self-care. Horizons emerged when the participants were asked to describe how the idea of manhood influences a man's life and health (Tables $1 \& 4)$.

Manhood and influences on life. Older MA men described manhood as a concept indicative of individuality and uniqueness. One participant stated:

"I think it has to do with their own self-definition of what manhood is. I don't think it follows any traditional practice, or for me it doesn't." 
Another participant stated:

"We make our own definition of how we are going to live every day, and that changes constantly. It isn't static."

Participants reported various levels of influence that helped develop and evolve their ideas of manhood. As the older MA male aged, certain levels of influence were described as more or less influential and impactful to their lives. One participant shared:

"I discovered early in life that being a role model is very important, and being the first born, there are certain responsibilities that come along with being a first-born. I had to do the best. I had to be the best. There was a lot of pride as well, in accomplishing what I have been able to accomplish. Like having my college degree. I acculturated. I don't see that as being an issue. Never completely assimilated, although obviously, I am assimilated. As you are growing up the challenges you face. They make me who I am today. It was never about being Mexican that made me do things this way. You're a man. You have got to do it this way. It wasn't about your ethnicity. It was about being a man!"

Participants described manhood as a concept that is related to strength, hard work, and responsibility. One of the participants described manhood as being both a positive and negative influence in a man's life:

"It could be both, a positive and negative influence. I'd say the negative is eventually going to get to you, health wise. The consuming of the alcohol and beer and smoking and all that will eventually get to you. The positive part of it would be the camaraderie, but after a while, if you don't grow up, camaraderie is not going to be there all the time for you. So, you got to start thinking about what's ahead for you. Some guys never grow up and they are still doing it. How 
are they still alive? How the hell can they do it still? At 60, 70, and 80 years old they are still downing them like if they were still 20 years old. I don't get it."

Manhood and influences on health-seeking and self-care. Participants were asked to describe how the idea of manhood influences a man's health. They described an aspect of manhood as being responsible for their own wellness. One participant shared: “Am I not a man because I go to the doctor? That's ridiculous! If I don't feel good, I go to the doctor. Who is going to take care of me? I am, and I know my doctor knows what I need. He knows what to do. Yes, masculinity can get in the way of some men, but not me. I know some men who don't care for their health and their bodies, but I do. I go see my doctor when I need to. He tells me if I am sick, not sick, and what I need to do. My masculinity has never gotten in my way of seeing my doctor. If I am sick, I go! Those times when I have felt sick, which have been very few, I tell my wife and then we go see my doctor."

Wellness was further described as a duty to self and others. One participant stated:

"Because you are the breadwinner. Because you are the caretaker. Because you are responsible. You owe it to yourself and to your family to take care of yourself. I have always maintained my health. I go to my appointments and I take care of myself."

Interestingly, manhood and "machismo" (a concept that was introduced during the interviews by the participants) were expressed as two very different concepts. Manhood was described as "father", which was further described as "hard worker" and "places family first". Manhood was also expressed as "brother", which was further expressed as "hard worker".

Machismo was described as practices and behaviors that are not becoming of a man. The participants acknowledged the existence and consequences of the behaviors related to machismo but did not self-identify with the notion. One participant shared: 
"I think it's heavily influenced. Culturally, there are some ideas, some notions, of masculinity that tough, hard-drinking, controlling, just in charge of everything, and that was in film, in literature, in the book I am reading Pedro Parra is about a patriarch. Not indifferent but separated from feelings and emotions. So, the Mexican notion of machismo has had a huge influence on the culture and it plays out negatively in domestic violence, in the students that I have worked with, and the families that I work with. Sometimes it just depends on maybe on influences that you have. Environment. I made a choice to turn away from it."

Acculturation. Health-seeking behaviors and health information gathering was not related to culture in this group of older MA men; rather, health promotion was associated with relationships (e.g., wives and health care providers) and personal choices. One participant stated: "It's all the same for all men. A man is a man. We are not different. We are all the same."

Participants described a time when they were younger and didn't think about their health. They didn't associate this lack of attention to their health with being MA or even being a man; rather, they associated it with youth, strength, and overall wellness. Participants also stated they "didn't think about health" when they were young men. They were "strong and didn't get sick." One participant shared:

"When you are younger, you think that you can beat the world. Nothing fazes you. I get a cold and I take a couple aspirins, and you keep on going. Now, you get a cold and it kicks you in the ying ying! Our resistance is not the same as it was then. Although we consider ourselves healthy, we are not like when we are young."

However, participants also "thought about going to see a doctor" when they were young men but described being "too poor" to afford one or had "no insurance." One participant stated: 
"Most of it was home remedies that the moms and grandmas would know what to do. If you had a fever, you do this. I remember my grandfather used to say, the fever is a man's best friend. It will always tell you when you have an infection or something wrong. So, do not take a fever as a bad thing. It's a warning."

Composite textural description. The experience of being an older MA man is expressed as being responsible for your wellness. It is described as a duty to self and others. Healthy behaviors included regular exercise, monitoring diet, regular doctor and dental visits, and acknowledging acute and chronic illnesses. Manhood and machismo were expressed as two very different concepts. Machismo was described as practices and behaviors that are not becoming of a man. The participants acknowledged the existence and consequences of the behaviors related to the concept but did not self-identify with the notion.

Composite structural description. Manhood was described as being observed and learned mostly through fathers and brothers. Although the idea of manhood was expressed as a selfcreated definition that is developed with age and experience, the participants linked hard work, family, and respect for women as integral components of manhood. The participants indicated that machismo is present in the MA culture and is observed and learned from older MA male family members and friends. They did not witness machismo in their fathers, but that they observed it in some of their peers and brothers.

Textural-structural synthesis. The participants expressed individual definitions of manhood, which did not include any behaviors that are said to be linked to the concept of machismo or necessarily related to culture. They expressed a sense of pride and commitment when it came to making choices that positively affected their health. They observed and learned manhood through their fathers and brothers. 


\section{Discussion}

The methodology of this study invited older MA males to share cultural, social, environmental, and gender factors influencing health-seeking behaviors and health information gathering. Two major themes emerged: 1) top external sources heeded include women, health care providers, and the Internet, and 2) men regard inner prompts for self-determined self-care.

The study was guided by the SEM, which provided us insight as to the various levels of influence on health information gathering and health-seeking behaviors. Our findings indicate an interplay between intrapersonal, interpersonal, institutional, and community factors. Wives, health care providers, and the Internet played an integral role in promoting health behaviors (i.e., PA, diet, and health care visits). The results regarding women as health promoters, partially supports previous research with MA males. Sobralske (2006a) found that women and wives influence men's health care decision-making. Women and wives were described as primary care takers, who were sought out for advice and during health concerns. Sobralske, (2006a) also found that women are observers of men, who push men to seek health care when they believe it is necessary. Peak et al. (2010) found that husbands (i.e., Latino men between 18 and 70 years) and wives made health-promoting decisions together. Our study and the Peak et al. findings were similar in that many of the participants described a partnership with their spouse (2010). The wives were regarded as an important component to health care decision- making. These findings support future health promotion programs that involve older MA dyads.

Our findings highlight the trusting relationship and valuable health promoting contributions made by health care providers among older MA men. Similarly, Vega, Rodriguez, \& Gruskin (2009) reported that health care providers play a vital role in increasing awareness, managing health behaviors, and motivating Latinos to be screened for diseases such as heart 
disease and diabetes. However, Hawkins et al. (2017) found that older Latino and AA males have a need to maintain control over their actions, presenting a barrier to health-seeking. Control can impede the ability to accept health advice from family and health care providers (Hawkins et al., 2017, p. 352). Rivera-Ramos \& Buki (2011) reported similar findings in their cohort of Latino men (mean age 54 years). Health promotion and risk reduction through preventive care, such as prostate cancer screening, transfers control to the health care provider and implies vulnerability (Rivera-Ramos \& Buki, 2011). It would be worth further investigating the relationship between the social construct of gender, age, ethnicity, socioeconomic status, education level, and geography among older MA men. This information may assist health care professionals adjust their health promotion and risk reduction methods to better serve the MA male population.

Camaraderie was a term used by the older MA men to describe the positive influence of manhood on a man's life. Houle et al. (2017) found that men with the highest health promotion behaviors were surrounded by inspirational role models. Houle at al. suggests that performing health behaviors with peers is a powerful facilitator and that male-only interventions are appealing to many men (2017). This interpersonal level of influence may be important to consider when developing health promotion interventions for older MA males.

We found that the older MA men refer to the Internet when gathering information on health. However, Valera, Lian, Brotzman, \& Reid (2018) reported that $79.13 \%$ of the minority men (i.e., 47.83\% Hispanic) in their study never searched for health information on the Internet. It's important to mention that $60.87 \%$ of the minority men did not currently have access to social media sites (Valera et al., 2018). In addition, only $15.65 \%$ of the participants were aged $55-70$ years old and the participants were formerly incarcerated (Valera et al., 2018). In 2012, 53\% of 
US older adult population reported using the Internet with $70 \%$ of them using it on a daily basis (Zickuhr \& Madden, 2012). Our findings support the use of technology for prompting health among older MA men. It is also worth investigating how correctional facilities may safely integrate this technology into prisons for the promotion of health in diverse populations of men.

Our analysis determined that the idea of manhood influences a man's life and health. The men described manhood, life, and health (good or bad) as concepts that grow and change with time. These concepts were expressed as different for everyone and not based on any one practice or belief. Similarly, Houle et al. (2017) found that comorbidities and age were positively associated with health responsibility. As men aged and health changed, they became more informed about health issues. We also found that health promotion was linked to personal choices and relationships with family members and health care providers, rather than culture. Our participants spoke of men being men with similarities that span beyond race and ethnicity. Likewise, Hawkins et al. (2017) found that both older Latino and AA men shared similar values and beliefs regarding what they learned about being a man growing up and what influenced health behaviors (p. 349). Similarly, Apesoa-Varano, Barker, Unutzer, \& Hinton (2015) reported that despite ethnic differences between White non-Hispanic and Mexican-origin older men, the men reported being united through shared ideas of manhood, such as being a hard worker and family provider. Future research may focus on examining perceptions of manhood and masculinity among older men in general to determine the direction for future health promotion interventions focused on decreasing men's health disparities.

Men in our study spontaneously mentioned machismo. However, as older MA men, they did not currently identify with the construct; therefore, it was not viewed as a barrier to health promotion behaviors. Interestingly, Arciniega, Anderson, Tovar-Blank, \& Tracey (2008) 
reported that young MA men endorse higher levels of traditional machismo (i.e., aggressive, sexist, chauvinistic, and hypermasculine behaviors), compared to older MA men. Similarly, participants in our study associated machismo specifically with youth and consuming large amounts of alcohol, smoking, not doing any female type work (e.g., making food at home), being in control of everything and everyone, and staying out late with their compadres. However, Getrich et al., (2012) found that older Hispano men (descendants of pure-blooded Spanish conquistadors) described machismo as a historical phenomenon that is no longer a problem (p. 10). Results from our study suggests that machismo can change over the life course and future health promotion interventions should be tailored toward MA men with consideration to differences in age. Similarly, Rivera-Ramos \& Buki (2011) suggests educating Latino men about the importance of preventive care at a young age to decrease the chances of developing negative attitudes that may be problematic in the future.

Limitations. There are possible limitations to the generalizability of these data. These data involved older MA males aged 65 years and older. Younger MAs may differ in their perspectives. This study was also conducted in Tucson, Arizona and attitudes may differ in other geographical locations. Despite the recruitment screening process and stratification by language preference or capability, many participants reported through the ARSMA-II subscales as mostly speaking and reading English at home. Furthermore, four of the monolingual Spanish participants struggled, trying to answer some of the interview questions in English. We are uncertain as to why these participants attempted to answer in English; however, social desirability (Polit \& Beck, 2017) may have influenced how participants approached answering. This phenomenon may or may not have impacted this study's findings. In addition, snowball sampling during recruitment may have resulted in selection bias. Lastly, a mid to high range US 
acculturation status among the participants may not reflect the lived experiences of all older MA men in the US. Regardless of these limitations, this study found that older MA males have specific perceptions of health and illness, health care practices, and beliefs about manhood that must be considered when developing evidence-based health promotion interventions focused on addressing men's health disparities.

\section{Implications for Future Research}

The Internet was a preferred source of health knowledge for these older MA males. Therefore, an evidence-based health promotion program may consider using a web-based or other technology-based approach to compliment a face-to-face intervention. The intervention should also involve participants and their wives or other types of trusted social support, such as a healthcare provider or peer, who may provide intervention content or serve as a spokesperson. Efforts should focus on promoting health behaviors through social networks. It would be worth investigating these interpersonal relationships to gain knowledge that can be applied to health promotion programs for older MA men and women.

Another implication that should be considered in future studies is classifying Hispanic men into subgroups according to race, age, ethnicity, socioeconomic status, and nativity. Expanding our knowledge will increase the opportunity to identify and appropriately address the health disparities and unique factors experienced by each subgroup (Aguirre-Molina, Borrell, \& Vega, 2010; Garcia et al., 2018; Getrich et al., 2012; Lampe, 2013; Vega et al., 2009).

Marketing strategies for recruitment of older MA men into an evidence-based health promotion program may also focus on the concept of self-care and its relationship to the concept of manhood. In our study, participants described caring for their health as a responsibility to themselves, their families, and their community. Our provides details on manhood and aging 
which may be relevant when working with older MA males in health promotion and risk reduction efforts. Future research should examine the association between traditional gender roles of manhood with health beliefs and health behaviors among MA males. Investigating this relationship through the life course may expand our understanding of the influence manhood may have on health behaviors at varying stages of a MA males life. Health promotion interventions may then be developed based on the mediating mechanisms of health behaviors and the unique health problems related to various definitions of manhood among MA men.

The relationship between acculturation, age, health behaviors, and gender in older MA men is also unclear. Previous studies reported that highly acculturated MAs are less healthy (Gorman, Read, \& Krueger, 1020). Future research must explore this relationship, discover its roots, and develop health promotion interventions specific to older MA males.

\section{Conclusion}

Fourteen older MA males participated in this one-of-a-kind phenomenological study. Interviews resulted in various levels of influence on health-seeking behaviors and health information gathering. Two major themes were found: 1) top external sources heeded include women, health care providers, and the Internet and 2) men regard inner prompts for selfdetermined self-care. The results of this study have increased the understanding of healthseeking behaviors and health information gathering in older MA males. However, there is still much we don't understand about health disparities and social determinants of health in older MA males. To identify problems, target resources, and design targeted interventions, reliable data must be gathered on this understudied and undeserved population.

\section{Acknowledgements}


The authors thank the generous men who allowed us into their homes and hearts and shared their experiences. We are grateful for your time and honored that you trusted us with your stories. We also thank Mrs. Larissa Airy Valenzuela Buelna. Gracias amigia. Sin ti, nada de esto habria sido posible. 
Table 1. Standardized Moderator's Guide: Interview Questions

\section{General Health}

1. Think of someone you consider healthy. Describe that person. What makes that person healthy?

2. How do you maintain your health?

3. Where do you gather your information about health?

a. Place

b. Person

c. Source

4. Who do you talk to about your health?

5. How does the idea of manhood influence a man's life?

6. How does the idea of manhood influence health?

\section{Masculinity}

1. How does the idea of manhood influence a man's life? How does manhood differ for a Mexican-American man as compared to a man of a different ethnicity?

a. How does manhood differ for a young Mexican-American man as compared to an older Mexican-American man?

b. How is the idea of manhood influenced by the Mexican-American culture or traditions?

c. How does the idea of manhood influence health? 


\section{Table 2. Participant Demographic Characteristics $(n=14)$}

\begin{tabular}{|c|c|c|}
\hline Demographic Variable & $\%(n)$ & Mean (SD) \\
\hline Older MA male's age (years) & & $71.2(4.5)$ \\
\hline Number of people in household & & $2.2(.89)$ \\
\hline \multicolumn{3}{|l|}{ Household } \\
\hline Lived alone & $7.1(1)$ & \\
\hline Lived with wife or partner & $78.6(11)$ & \\
\hline Lived with children & 0 & \\
\hline Lived with grandchildren & 0 & \\
\hline Lived with friends & 0 & \\
\hline Lived with other family members & $14.3(2)$ & \\
\hline \multicolumn{3}{|l|}{ Marital Status } \\
\hline Married & $92.9(13)$ & \\
\hline Never married & 0 & \\
\hline Separated & 0 & \\
\hline Divorced & 0 & \\
\hline Widowed & $7.1(1)$ & \\
\hline \multicolumn{3}{|l|}{ Education Level } \\
\hline Never attended school & 0 & \\
\hline Elementary school (K through 6$)$ & 0 & \\
\hline Junior high (grades 7 and 8 ) & $14.3(2)$ & \\
\hline Some high school & 0 & \\
\hline High school graduate & $21.4(3)$ & \\
\hline GED & 0 & \\
\hline Some college/vocational training & $21.4(3)$ & \\
\hline Trade school/vocational training graduate & $7.1(1)$ & \\
\hline College graduate & $7.1(1)$ & \\
\hline Graduate school & $28.6(4)$ & \\
\hline \multicolumn{3}{|l|}{ Language Spoke Mostly at Home } \\
\hline English & $78.6(11)$ & \\
\hline Spanish & $21.4(3)$ & \\
\hline \multicolumn{3}{|l|}{ Language Read Mostly at Home } \\
\hline English & $78.6(3)$ & \\
\hline Spanish & $21.4(3)$ & \\
\hline \multicolumn{3}{|l|}{ Paying Job } \\
\hline Yes & $14.3(2)$ & \\
\hline No & 0 & \\
\hline Retired & $85.7(12)$ & \\
\hline
\end{tabular}


Household Income Per Year

More than $\$ 25,000$

$78.6(11)$

$\$ 20,000$ - \$25,000

$14.3(2)$

$\$ 15,000$ - $\$ 20,000$

0

$\$ 10,000$ - $\$ 15,000$

$7.1(1)$

Less than $\$ 10,000$

0

ARSMA-II

AOS

$3.92(.19)$

MOS

$3.42(.28)$ 
Table 3. Select Quotes of Emerging Theme: Top Outside Sources Heeded Include Women, Health care Providers, and the Internet

\section{Women}

- My wife is important in my health and health decisions.

- She is my constant companion.

- I think women are more concerned and interested in people seeing a doctor, whenever necessary, or even when not necessary. They also help us keep appointments. Women have always done health more than men. It's always been like this. They do way more things when it comes to health. My wife is a believer that if something is wrong, go to the doctor. You know sometimes you may have something wrong for a while, but until it really hits us, then it's time to go to the doctor.

- I used to avoid going to see the doctor. I didn't feel like I had to go see the doctor and I think one of the reasons I changed was my wife. She stayed on me to get my physical and see my doctor. She prodded me to go.

- Her (i.e., wife) diet has influenced my diet. I have diabetes type two. When I found that out back in 1995, I had to change my ways also in terms of eating and it has been a struggle for me, but she has always been there telling me what I can and cannot do.

\section{Health Care Providers}

- I trust him.

- I have been with her for many years.

- I have good discussions with him.

- I am comfortable with him.

- He motivates me.

- He calls me, not his staff.

- He is current on research and technology.

- He gives out his cellphone number to his patients.

\section{Internet}

- I read a lot of stuff on the Internet, so that kind of helps me out.

- It's funny, now I go to the Internet (laughing).

- Once in a while, if I have a question regarding health, I go to the Internet and look it up or else anytime I see a little booklet or pamphlet that kind of sounds like a subject I'm interested in.

- Well, right now it's just the information that is on the Internet. It's at your fingertips.

- Besides my wife, I will look up stuff on the website. Things that maybe I am concerned about or things that I am not aware of, or if I read something, I will follow up there.

- I go to websites. Although I hate relying on those because you know of misinformation and how it gets written wrong. I might look things up and bring it up to my doctor as to what he thinks, and get his opinion. 
Table 4. Select Quotes of Emerging Theme: Men Regard Inner Prompts for Self-Determined Self-Care.

\section{Manhood and Influences on Life}

- I think it has a lot to do with their own self-definition of what manhood is. I don't think it follows any traditional practice, or for me it doesn't.

- I think ideas of manhood influence your choices, and a choice could be disastrous.

- We make our own definition of how we are going to live every day, and that changes constantly. It isn't static.

- I think manhood is very individual.

- If you are a man, you are considered to be somewhat strong.

- Manhood makes you work harder, because you have to. You have responsibilities.

- I worked hard because I wanted to be known as the best worker.

- You came home tired because you gave it your all. It didn't matter what you did because you gave it your all because you were not going to let it be said that you failed. You didn't fail as a man.

- It could be both, a positive and negative influence. I'd say the negative is eventually it's going to get to you, health wise. The consuming of the alcohol and beer and smoking and all that will eventually get to you.

- Changing your manhood is becoming more enlightened of what is out there that can help you as an individual.

\section{Manhood and Influences on Health}

- I don't have a problem seeing a doctor.

- I will go to the doctor. I have no issues with that. I am a man and I go see my doctor. If I feel bad, I go. I have always been that way. I need my health.

- Because you are the breadwinner. Because you are the caretaker. Because you are responsible. You owe it to yourself and to your family to take care of yourself.

- Am I not a man because I go to the doctor? That's ridiculous! If I don 't feel good, I go to the doctor. Who is going to take care of me? I am, and I know my doctor knows what I need. He knows what to do. Yes, masculinity can get in the way of some men, but not me. I know some men who don't care for their health and their bodies, but I do. I go see my doctor when I need to. He tells me if I am sick, not sick, and what I need to do. My masculinity has never gotten in my way of seeing my doctor. If I am sick, I go! Those times when I have felt sick, which have been very few, I tell my wife and then we go see my doctor.

- You were never explained that going to the doctor was good.

- I remember my Dad. He wouldn't go to the hospital. He wouldn't take medications. He smoked a pipe. I never heard him say he was sick. He never complained. Was it manhood, or was it just him? Nothing got to him.

- I try but don't always agree with the doctor, when he says come in three months. Oh no! That is too quick. Put it for six months or once a year. Unless I am sick, I don't need to go. 


\section{References}

Adler, N.E., Cutler, D.M., Fielding, J.E., Galea, S., Glymour, M.M., Koh, H.K., \& Satcher, D. (2016). Addressing social determinants of health and health disparities: a vital direction for health and health care. Retrieved from https://nam.edu/wp-content/uploads/2016/09/Addressing-SocialDeterminants-of-Health-and-Health-Disparities.pdf

Aguirre-Molina, M., Borrell, L.N., \& Vega, W. (2010). Health Issues in Latino Males. A social and structural approach. New Jersey: Rutgers University Press.

Ailinger, R. L. (1989). Self-assessed health of Hispanic elderly persons. Journal of Community Health Nursing, 6(2), 113-118.

Apesoa-Varano, E.C., Barker, J.C., Unutzer, J., \& Hinton, L. (2015). Depression attributes among White non-Hispanic and Mexican-origin older men. American Journal of Geriatric Psychiatry, 23(9), $960-969$.

Arciniega, G.M., Anderson, T.C., Tovar-Blank, Z.G., \& Tracey, T.J.G. (2008). Toward a fuller conception of machismo: development of a traditional machismo and caballerismo scale. Journal of Counseling Psychology, 55(1), 19-33.

Baker, P. (2018). Men's health: time for a new approach. Physical Therapy Reviews, 23(2), $144-150$.

Centers for Disease Control and Prevention (CDC). (2013). The state of aging and health in America 2013. Retrieved from http://www.cdc.gov/aging/pdf/state-aging-health-in-america-2013.pdf

Centers for Disease Control and Prevention (CDC). (2013a). Division for heart disease and stroke prevention. Men and heart disease fact sheet. Retrieved from http://www.cdc.gov/DHDSP/data_statistics/fact_sheets/fs_men_heart.htm

Courtenay, W.H. (2011). Dying to be men. Psychosocial, environmental, and behavioral directions in promoting the health of men and boys. New York: Routledge. 
Creswell, J.W. (2009). Research design: qualitative, quantitative, and mixed methods approaches (SAGE South Asia edition) ( $3^{\text {rd }}$ ed.). South Asia: SAGE Publications, Inc.

Creswell, J.W. (2013). Qualitative inquiry \& research design. Choosing among five approaches. Los Angeles, CA: SAGE Publications, Inc.

Cuellar, I., Arnold, B., \& Maldonado, R. (1995). Acculturation rating scale for Mexican Americans-II: a revision of the original ARSMA scale. Hispanic Journal of Behavioral Sciences, 17(3), 275-304.

Espino D.V., Macias R.L., Wood R.C., Becho J., Talamantes M., Finley, M.R., Hernandez A.E., \& Martinez R. (2010). Physician-assisted suicide attitudes of older Mexican-American and nonHispanic white adults: does ethnicity make a difference? JAGS 58(7), 1370-1375.

Fenelon, A., Chinn, J.J., \& Anderson, R.N. (2017) A comprehensive analysis of the mortality experience of Hispanic subgroups in the United States: variation by age, country of origin, and nativity. SSM Population Health, 3, $245-254$.

Field, C.A., Cabriales, J.A., Woolard, R.H., Tych, A.H., Caetano, R. \& Castro, Y. (2015). Cultural adaptation of a brief motivational intervention for heavy drinking among Hispanics in a medical setting. BioMed Central Public Health, 15(724), 1 - 12.

Friedman, Hooker, Wilcox, Burroughs, \& Rheaume, (2012). African American men's perspectives on promoting physical activity: we're not that difficult to figure out! Journal of Health Communication 17(10), 1151-1170.

Garcia, M.A., Garcia, C., Chiu, CT., Raji, M., \& Markides, K.S. (2018). A comprehensive analysis of morbidity life expectancies among older Hispanic subgroups in the United States: variation by nativity and country of origin. Innovation in Aging, 2(2), $1-12$. 
Getrich, C.M., Sussman, A.L., Helitzer, D.L., Hoffman, R.M., Warner, T.D., Sanchez, V., Solares, A., \& Rhyne, R.L. (2012). Expressions of machismo in colorectal cancer screening among New Mexico Hispanic subpopulations. Qualitative Health Research, 22(4), 546 - 559.

Gilbert, P.A., Barrington, C., Rhodes, S.C., \& Eng, E. (2016). Saliendo adelante: stressors and coping strategies among immigrant Latino men who have sex with men in a nontraditional settlement state. American Journal of Men's Health, 10(6), 515 - 525.

Gold, M. (2014). Reducing health care disparities: where are we now? Retrieved from http://www.rwjf.org/content/dam/farm/reports/issue_briefs/2014/rwjf411511

Gorman, B.K., Read, J.G., \& Krueger, P.M. (2010). Gender, acculturation, and health among Mexican Americans. Journal of Health and Social Behavior, 51(4), 440 - 457.

Hawkins, J., Watkins, D.C., Kieffer, E., Spencer, M., Piatt, G., Nicklett, E.J., Lebron, A., Espitia, N., \& Palmisano, G. (2017). An exploratory study of the impact of gender on health behavior among African American and Latino men with type 2 diabetes. American Journal of Men's Health, $11(2), 344-356$.

Hill, T.D., Uchino, B.N., Eckhardt, J.L., \& Angel, J.L. (2016). Perceived social trajectories and the allcause mortality risk of older Mexican American women and men. Research on Aging, 38(3), $374-398$.

Houle, J., Meunier, S., Coulombe, S., Mercerat, C., Gaboury, I., Tremblay, G., de Montigny F., Cloutier, L., Roy, B., Auger, N., \& Lavoie, B. (2017). Peer positive social control and men's healthpromoting behaviors. American Journal of Men's Health, 11(5), 1569 - 1579.

Institute of Medicine (IOM). (2009). Race, ethnicity, and language data: standardization for health care quality improvement. Washington, DC: National Academies Press.

Lampe, P.E. (2013) Mexican Americans: a sociological introduction. Retrieved 
from http://www.uiw.edu/hass/sociology//documents/lampebookonlineversion613.pdf.

Leone, J.E., Rovito, M.J., Mullin, E.M., Mohammed, S.D., \& Lee, C.S. (2017). Development and testing of a conceptual model regarding men's access to health care. American Journal of Men's Health, $11(2), 262-274$.

Masel, M.C., Rudkin, L.L., \& Peek, M.K. (2006). Examining the role of acculturation in health behaviors of older Mexican Americans. American Journal of Health Behavior, 30(6), 684 - 699.

McEwen, M.M \& Murdaugh, C. (2012). Demographic data form. University of Arizona College of Nursing, Family Diabetes Study.

Moustakas, C. (1994). Phenomenological research methods. Thousand Oaks, CA: Sage Publications, Inc.

National Center for Health Statistics (NCHS). (2010). United States life tables by Hispanic origin. Retrieved from http://www.cdc.gov/nchs/data/series/sr_02/sr02_152.pdf

Noone, J.H. \& Stephens, C. (2008). Men, masculine identities, and health care utilisation. Sociology of Health \& Illness, 30(5), $711-725$.

Nordstrom, A., Hadrevi, J., Ollsson, T., Franks, P.W., \& Nordstrom, P. (2016). Higher prevalence of type 2 diabetes in men than in women is associated with differences in visceral fat mass. Journal of Clinical Endocrinology and Metabolism, 101(10), 3740-3746.

Olsen, S. \& Anderson, K.M. (2018). Immigration as a social determinant of health: proceedings of a workshop. Washington, DC: The National Academies Press.

Patton, M.Q. (2002). Qualitative research \& evaluation methods (3 ${ }^{\text {rd }}$ ed.). Thousand Oaks, CA: Sage Publications, Inc.

Peak, T., Gast, J., \& Ahlstrom, D. (2010). A needs assessment of Latino men's health concerns. American Journal of Men's Health, 4(1), 22-32. 
Perkins, H. S., Hazuda, H. P., \& Cortez, J. D. (2004). Advance care planning: does patient gender make a difference? The American Journal of the Medical Sciences, 327(1), 25-32.

Perkins, H.S., Cortez, J.D., \& Hazuda, H.P. (2006). Advance care planning: does patient gender make a difference? The American Journal of the Medical Sciences, 327(1), 25 - 32.

Polit, D.F., \& Beck, C.T. (2017). Nursing research: generating and assessing evidence for nursing practice $\left(10^{\text {th }}\right.$ ed.). Philadelphia, PA: Wolters Kluwer.

Rivera-Ramos, Z.A. \& Buki, L.P. (2011). I will no longer be a man! Manliness and prostate cancer screenings among Latino men. Psychology of Men \& Masculinity, 12(1), 13 - 25.

Salinas, J., McCormick, J.B., Rentfro, A., Hanis, C., Hossain, M.M., \& Fisher-Hoch, S.P. (2011). The missing men: high risk of disease in men of Mexican origin. American Journal of Men's Health, $5(4), 332-340$.

Sallis, J.F., Owen, N., \& Fisher, E.B. (2008). Ecological models of health behavior. In K. Glanz, B.K. Rimer, \& K. Viswanath (Eds), Health behavior and health education: theory, research, and practice $\left(4^{\text {th }}\right.$ ed.) (pp. $\left.465-485\right)$. San Francisco, CA: Jossey-Bass.

Sobralske, M.C. (2006). Community-based strategies to improve the health of Mexican American men. International Journal of Men's Health, 5(2), 153 - 171.

Sobralske, M.C. (2006a). Health care seeking among Mexican American men. Journal of Transcultural Nursing, 17(2), $129-138$.

Stimpson, J.P. \& Peek, M.K. (2005). Concordance of chronic conditions in older Mexican American couples. Preventing Chronic Disease: Public Health Research, Practice, and Policy, 2(3), 1 - 7. Stokols, D. (1992). Establishing and maintaining healthy environments. American Psychologist, 47(1), 6-22. 
Thorpe, R.J., Richard, P., Bowie, J.V., LaVeist, T.A., \& Gaskin, D.J. (2013). Economic burden of men's health disparities in the United States. International Journal of Men's Health, 13(3), 195 - 212.

United Nations Population Fund (UNFPA). (2012). Ageing in the twenty-first century: a celebration and a challenge. New York, NY: United Nations Population Fund.

Valera, P., Lian, Z., Brotzman, L., \& Reid, A. (2018). Fatalistic cancer beliefs and information seeking in formerly incarcerated African-American and Hispanic men: implications for cancer health communication and research. Health Communication, 33(5), 576 - 584.

Vega, W.A., Rodriguez, M.A., \& Gruskin, E. (2009). Health disparities in the Latino population. Epidemiologic Reviews, 31, 99-112.

Watt, G.P., Vatcheva, K.P., Griffith, D.M., Reininger, B.M., Beretta, L., Fallon, M.B., McCormick, J.B., \& Fisher-Hoch, S.P. (2016). The precarious health of young Mexican American men in south Texas, Cameron county Hispanic cohort, 2004 - 2015. Preventing Chronic Disease: Public Health Research, Practice, and Policy, 13(E113), 1 - 13.

Zickuhr, K. \& Madden, M. (2012). Older adults and internet use. Retrieved from http://www.pewinternet.org/2012/06/06/older-adults-and-internet-use/

Zong, J., Betalova, J. \& Hallock, J. (2018). Frequently requested statistics on immigrants and immigration in the United States. Retrieved from https://www.migrationpolicy.org/article/frequently-requested-statistics-immigrants-andimmigration-united-states 